
with Controlled Helical Structures for $\mathrm{THz}$

SUBJECT AREAS:

METAL-ORGANIC

FRAMEWORKS

MATERIALS FOR DEVICES

STRUCTURAL MATERIALS

PHOTONIC DEVICES

Received

6 December 2013

Accepted

22 April 2014

Published

12 May 2014

Correspondence and requests for materials should be addressed to K.K. (kamata.k.ab@m. titech.ac.jp); T.I. (iyoda.t.aa@m.titech. ac.jp) or M.H. (hangyo@ile.osaka-u. ac.jp)

\section{Electromagnetic Responses}

Kaori Kamata ${ }^{1,2}$, Zhenzi Piao ${ }^{1}$, Soichiro Suzuki ${ }^{3}$, Takahiro Fujimori ${ }^{3}$, Wataru Tajiri ${ }^{3}$, Keiji Nagai ${ }^{1,3}$, Tomokazu lyoda 1,2,3, Atsushi Yamada 2,4 Toshiaki Hayakawa ${ }^{2,5}$, Mitsuteru Ishiwara ${ }^{6}$, Satoshi Horaguchi ${ }^{6}$, Amha Belay7, Takuo Tanaka ${ }^{2,8}$, Keisuke Takano9 \& Masanori Hangyo 9

'lyoda Supra-Integrated Material Project, Exploratory Research for Advanced Technology (ERATO), Japan Science and Technology Agency (JST), 4259 Nagatsuta-Cho, Midori-Ku, Yokohama, Kanagawa 226-8503, Japan, ${ }^{2}$ Research Society for Biotemplate Technology, 4259 Nagatsuta-Cho, Midori-Ku, Yokohama, Kanagawa 226-8503, Japan, ${ }^{3}$ Division of Integrated Molecular Engineering, Chemical Resources Laboratory, Tokyo Institute of Technology, 4259 Nagatsuta-Cho, Midori-Ku, Yokohama, Kanagawa 226-8503, Japan, ${ }^{4}$ Technology and Engineering Department, Materials Division, Sumitomo Metal Mining Co. Ltd., $5-$ 11-3 Shimbashi, Minato-Ku, Tokyo 105-8716, Japan, ${ }^{5}$ Advantest Laboratories Ltd., 48-2 Matsubara, Kami-Ayashi, Aoba-Ku, Sendai, Miyagi 989-3124, Japan, ${ }^{6}$ DIC LIFETEC Co., Ltd., 12 Yawata-Kaigandori, Ichihara, Chiba 290-8585, Japan, ${ }^{7}$ Earthrise Nutritionals, LLC., 113 E. Hoober Road Calipatria, CA 92233, USA, ${ }^{8}$ Metamaterials Laboratory, Advanced Science Institute, RIKEN, Wako, Saitama 351-0198, Japan, Institute of Laser Engineering, Osaka University, 2-6 Yamadaoka, Suita, Osaka 565 0871, Japan.

Microstructures in nature are ultrafine and ordered in biological roles, which have attracted material scientists. Spirulina forms three-dimensional helical microstructure, one of remarkable features in nature beyond our current processing technology such as lithography in terms of mass-productivity and structural multiplicity. Spirulina varies its diameter, helical pitch, and/or length against growing environment. This unique helix is suggestive of a tiny electromagnetic coil, if composed of electro-conductive metal, which brought us main concept of this work. Here, we describe the biotemplating process onto Spirulina surface to fabricate metal microcoils. Structural parameters of the microcoil can be controlled by the cultivation conditions of Spirulina template and also purely one-handed microcoil can be fabricated. A microcoil dispersion sheet exhibited optically active response attributed to structural resonance in terahertz-wave region.

$\mathrm{P}$ eople have been fascinated by highly complex microstructures produced in nature ${ }^{1}$. Natural materials are sophisticatedly organized in response to specific functions, whose systematical organizing processes are superior to any engineering routes for smart materials in the light of multiplicity, optimal integration, and low energy consumption. In naturally occurring microstructures, important feature is their high dimensionality, which stimulates material scientists to design functional materials. Biomimetics or bioinspiration ${ }^{2-4}$, learning microstructures produced in nature, have drawn attention as one of breakthroughs on material science and processing. But profound understanding of formation mechanism established under natural selection process must be required to develop the biomimetic process. In fact, we have suggested collaborating with nature, i.e., biotemplating process, which directly borrows the natural microstructures for new material fabrications (Fig. 1). A concept of this process can lead to mass productivity with less energy. Main issue is how to control the natural microstructures. If we can find effective factors controlling the structures from various environmental conditions, the biotemplating process would have unlimited potential and will be global strategy for material development. We have focused three-dimensional helical feature in nature as biotemplate candidate ${ }^{5}$. Helical microalgae, Spirulina (Arthrospira platensis) ${ }^{6,7}$ naturally shaping left-handed (LH) open helical structure and already commercialized as nutritional supplements or food materials ${ }^{8}$, was employed in this study. Its helical structure is very sensitive against the environmental conditions, which should be strong advantage to exploit a variety of diameter, pitch, and handedness. Development of biotemplating process using Spirulina can achieve mass production of microcoil ( $\mu$ coil), which is currently manufactured by precision machining or lithography one by one. Since Chen et al. first coated the Spirulina surface with magnetic ferrite ${ }^{9}$, several biotemplating processes using the Spirulina 


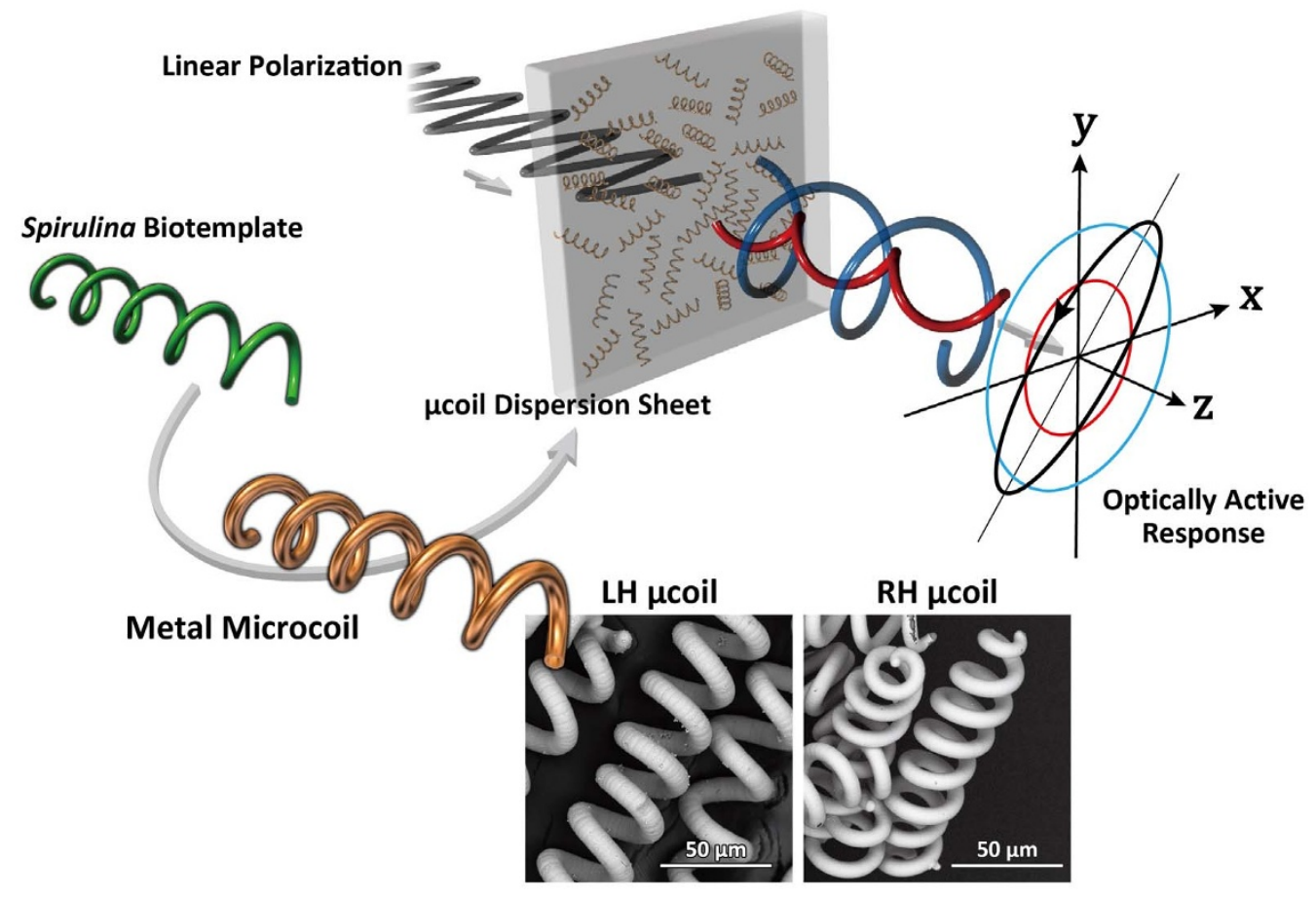

Figure $1 \mid$ Fabrication of Spirulina-templated microcoil ( $\mu$ coil) and its optical activity against THz wave. The Spirulina (Arthrospira platensis) was utilized as biotemplate to mass fabricate $3 \mathrm{D}$ helical $\mu$ coils. An enantiomeric pair of left-handed (LH) and right-handed (RH) $\mu$ coils can be fabricated with $100 \%$ of optical purities as SEM images show. The THz time-domain spectroscopies with polarimetric analyses (THz-TDS-PA) of paraffin sheets containing the $\mu$ coils were measured to examine optical activities and structural resonances based on the helical structures.

have been reported in the field of material science. However, no function specific to its helical microstructure has yet been discovered.

\section{Results}

Structure-controllability of Spirulina biotemplate. The LH helix of Spirulina is a common structure found in nature and easily obtained as the stock strain from culture collections. Under the conventional cultivation condition ${ }^{10}$, average feature size of the LH Spirulina (Fig. 2a) were $6 \mu \mathrm{m}$ in wire diameter $(d), 43 \mu \mathrm{m}$ in coil diameter $(D), 174 \mu \mathrm{m}$ in free length of coil $\left(L_{\text {free }}\right), 74 \mu \mathrm{m}$ in coil pitch $\left(L_{\text {free }} / N\right)$, 2.4 in turn number $(N)$, and $29^{\circ}$ in pitch angle $(\alpha)$ (for graphical illustration of the symbols, see Fig. S1). The number of Spirulina in $1 \mathrm{~mL}$ increased from $10^{2}$ to $10^{5}$ within one week (Methods and Fig. S2). During the cultivation, the $d, D$, and $L_{\text {free }} / N$ remained with narrow size distributions (around $5-15 \%$ of relative standard deviation, RSD), while the $L_{\text {free }}$ had a higher RSD around $20 \%$ because Spirulina grows along the long axis direction (Fig. S3). It has been known that the Spirulina forms various helical features and even linear shape under different environments. In this study, the $L_{\text {free }} / N$ was systematically controlled by varying cultivation temperature and light intensity (Methods and SI-II) ${ }^{11}$. The series of LH Spirulina with five different helical features in Fig. 2 are numbered as LH template-1 to -5. Linear Spirulina often found in laboratory cultivation of regular helical strains was obtained in pure culture (Fig. 2f). Such flexible morphologies against the environment can achieve distinction as microstructured-materials separately from commonly-used genetic control.

Spirulina-based biotemplating process. We have designed the biotemplating process using electroless plating technique, which can generate smooth metal layer selectively on surface of targeting object to be plated. In this study, the process includes (i) fixation of Spirulina, (ii) Pd catalyzation (Pd nanoparticle adsorption as plating catalyst nuclei ${ }^{12,13}$ ), and (iii) copper electroless plating (Methods, see also Supplementary SI-III). The process (i) employed general method of tissue fixation by glutaraldehyde, which cross-links amine groups of amino-acid side chains in proteins ${ }^{14}$. The process (ii) includes two steps with $\mathrm{Pd}$ ion adsorption and reduction of the Pd ion to form the metallic $\mathrm{Pd}$, which can work as catalyst oxidizing reducing reagent for the metal deposition from the plating bath. For the electroless plating, bath load, i.e., the total surface area of Spirulina to amount of plating bath, was adjusted to be $200 \mathrm{~cm}^{2} / \mathrm{L}$. In the laboratory scale with $1 \mathrm{~L}$ of plating bath, one batch from $20 \mathrm{~mL}$ of the Spirulina cultivation medium with $10^{5} \mathrm{~mL}^{-1}$ in Spirulina concentration gave approximately 2 million $\mu$ coils (90 mg, 80\% yield). The resulting $\mu$ coils were quantitatively and qualitatively characterized by ICP, XPS, $\mathrm{XRD}$, and EDX-SEM at different stage of biotemplating process (for the detailed information, see supplementary SI-III). It was found that the Pd catalyst was adsorbed to the surface as well as inside the tissue of Spirulina with almost $2 \mathrm{vol} \%$ in volume fraction to the Spirulina. The $\mu$ coil contained the metallic $\mathrm{Pd}$ of $>80 \%$ and a little amount of $\mathrm{PdO}_{\mathrm{x}}$ in the nanoparticles.

The copper layer deposited on the Spirulina was consisting of the metal (>90\% in content) with the oxides at the surface. The $\mu$ coils looked reddish brown with metallic luster and had tubular structure with around $550 \mathrm{~nm}$ in thickness, whose interior space still included the fixed Spirulina template (Fig. S15). The thickness can be controlled by changing the plating time and also the bath load, here, we demonstrated with $200 \mathrm{~cm}^{2} / \mathrm{L}$ in the bath load $(20$-mL loading amount) to obtain the thickness enough to exhibit bulk-like electric property. The efficiency of $\mathrm{Cu}$ deposition and relationship between the bath load and the thickness were discussed in supplementary SIIV. The optical micrographs of $\mu$ coils ( $\mathrm{LH} \mu$ coil-1 to -5) fabricated from corresponding LH template- 1 to -5 are shown in Figure $2 \mathrm{~g}-2 \mathrm{k}$ along with straight copper wire (Fig. 2l) from the linear strain. Emphasis should be placed on that the microstructures after the biotemplating processes were faithfully transferred from those of the Spirulina templates, for example, in case of LH $\mu$ coil-1, $d=$ $7.0 \mu \mathrm{m}(15 \% \mathrm{RSD}), D=41 \mu \mathrm{m}(14 \%), L_{\text {free }}=174 \mu \mathrm{m}(26 \%), L_{\text {free }} /$ $N=77 \mu \mathrm{m}(9 \%), N=2.3$, and $\alpha=31^{\circ}$ (see also Fig. S16). 


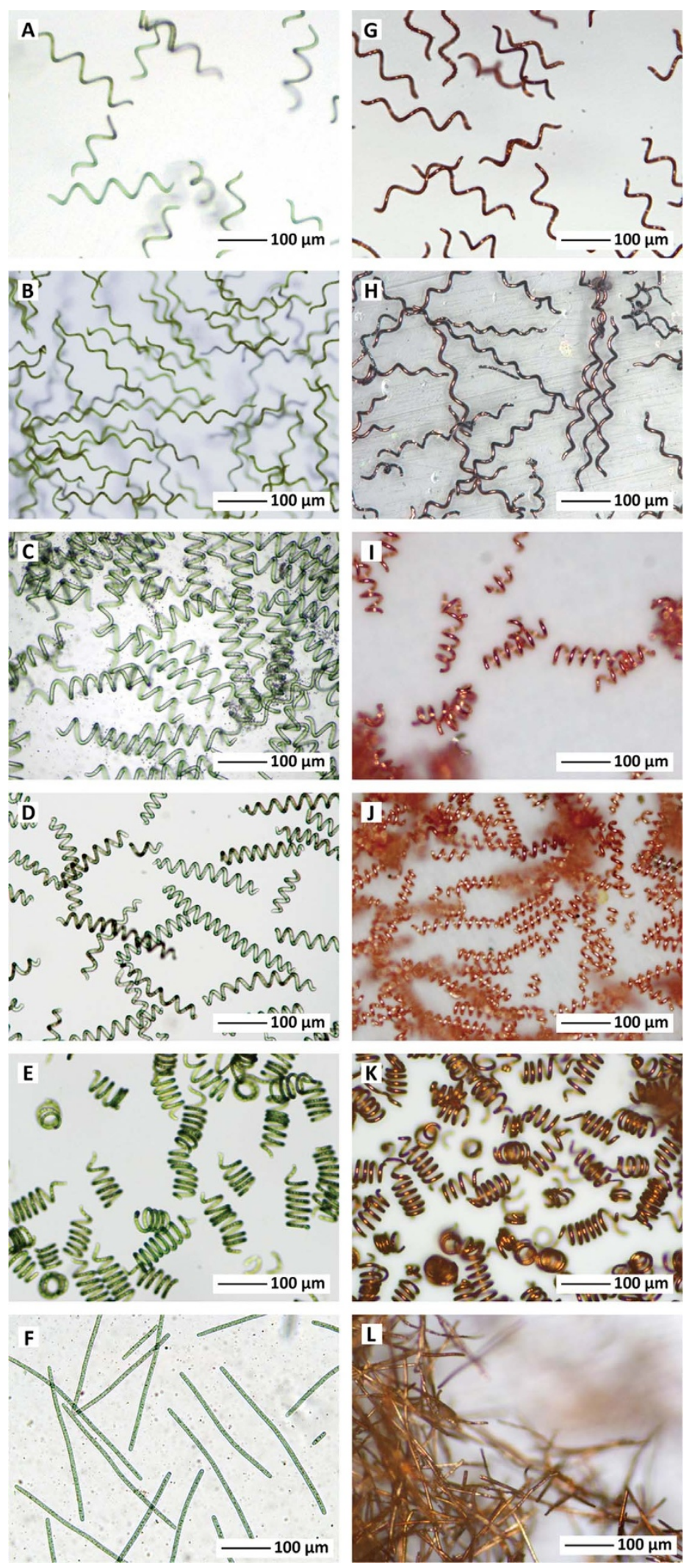

Figure $2 \mid$ Left-handed (LH) Spirulina-templated microstructures. The Spirulina basically forms LH helix but its structural parameters such as helical pitch, length, number of turns, and handedness are of variety based on a kind of strains. Sensitive strain, NIES-46, gradually tightens the helical pitch as the cultivation proceeded with stronger light intensity and higher temperature. The systematic adjustment of the helical pitch, $L_{\text {free }} / N$, can be demonstrated; (a)-(e), LH template-1 to -5. (f) Linear strain was prepared by the pure cultivation of laboratory-derived single trichome. The biotemplating process was successfully carried out to generate the copper $\mu$ coils whose structures are followed by those of LH templates: $\left(\right.$ coil number, $\left.L_{\text {free }} / N\right)$; $(\mathrm{g}) \mathrm{LH} \mu \mathrm{coil}-1$, $77 \mu \mathrm{m}$; (h) LH $\mu$ coil-2, $55 \mu \mathrm{m}$; (i) LH $\mu$ coil-3, $27 \mu \mathrm{m}$; (j) LH $\mu$ coil-4, $20 \mu \mathrm{m}$; (k) LH $\mu$ coil-5, $16 \mu \mathrm{m}$. (l) The straight copper wire was also properly templated from the linear strain. 

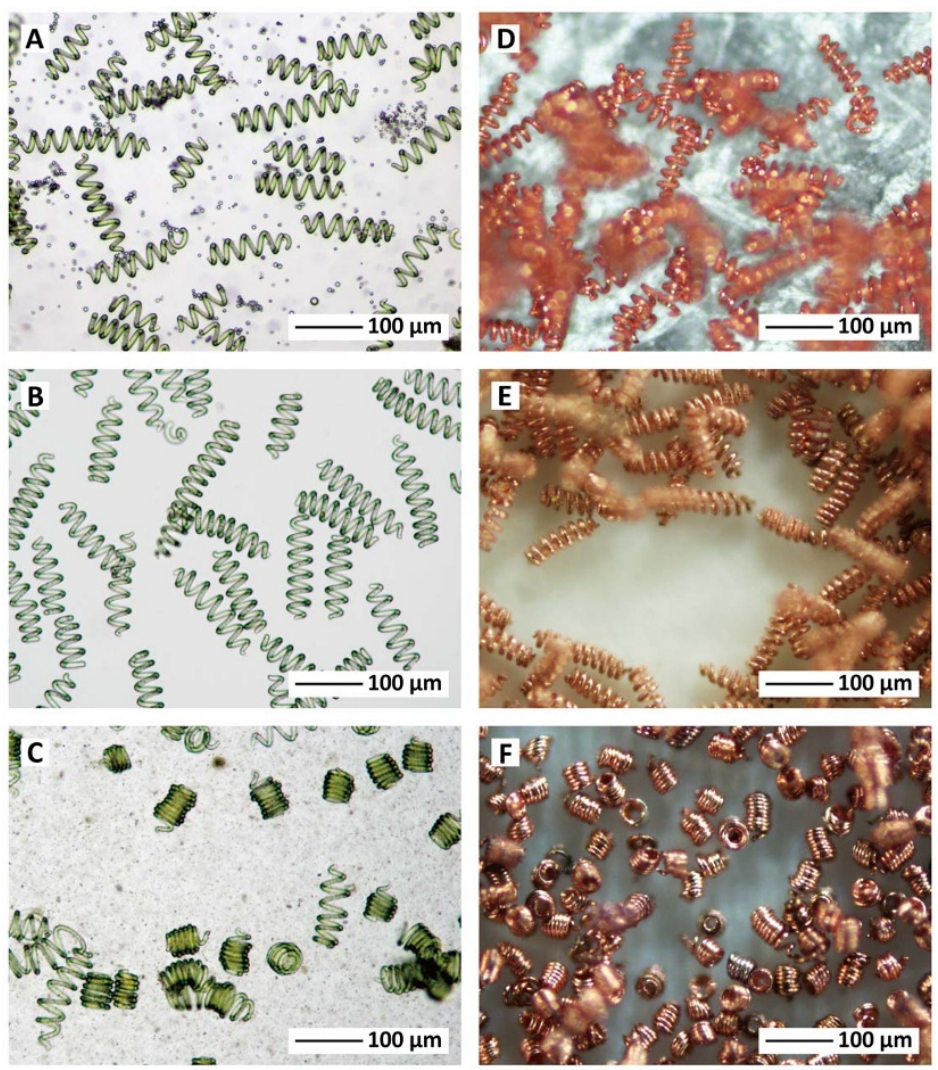

Figure 3 Right-handed (RH) Spirulina and their biotemplated products. RH template-1 to -3 with three different $L_{\text {free }} / N s$, (a) - (c), generated the corresponding RH $\mu$ coils: (coil number, $L_{\text {free }} / N$ ); (d) RH $\mu$ coil-1, $19 \mu \mathrm{m}$; (e) RH $\mu$ coil-2, $14 \mu \mathrm{m}$; (f) RH $\mu$ coil-3, $6 \mu \mathrm{m}$.

Right-handed (RH) strains of Spirulina are rare but they are found both in nature and in culture. Reversal of helical handedness from left to right and vice versa has been observed and ascribed to genetic drift or environmental factors like temperature uplift or mechanical stress $^{15}$. One such RH strain obtained from Earthrise Nutritionals, California, USA, was grown in pure culture from a single trichome. The RH Spirulina was more tightly coiled smaller than around $20 \mu \mathrm{m}$ in $L_{\text {free }} / N$. The RH templates and the corresponding $\mu$ coils were prepared in the same manner as the LH series (Fig. 3 and
Fig. S17). The structural parameters for all of $\mu$ coils and referenced samples are summarized in Table 1.

Can the $\mu$ coil behave as chiral electromagnetic material? We prepared dispersion silicon sheets of LH $\mu$ coil-1 for transmission and reflection spectroscopies in the region of millimeter wave with free space method (Fig. $4 \mathrm{a}$ and $4 \mathrm{~b}$, SI-VI). The logarithmic transmittance decreased as the concentration of $\mu$ coil was increased. Reflection components were almost constant against the

Table 1 | The geometric parameters of $\mu$ coil

\begin{tabular}{|c|c|c|c|c|c|c|c|c|c|c|}
\hline Parameters & $\begin{array}{c}\text { wire } \\
\text { diameter }\end{array}$ & $\begin{array}{c}\text { coil } \\
\text { diameter }\end{array}$ & $\begin{array}{l}\text { free length } \\
\text { of pitch }\end{array}$ & $\begin{array}{l}\text { number } \\
\text { of turn }\end{array}$ & $\begin{array}{c}\text { free length } \\
\text { of coil }\end{array}$ & $\begin{array}{l}\text { length of wire } \\
\text { for one pitch }\end{array}$ & $\begin{array}{l}\text { length of wire } \\
\text { for one coil }\end{array}$ & $\begin{array}{l}\text { pitch } \\
\text { angle }^{1}\end{array}$ & $\begin{array}{c}\text { theoretical } \\
\text { frequency } \\
\text { region }{ }^{2}\end{array}$ & $\begin{array}{l}\text { detected } \\
\text { frequency } \\
\text { region }^{3}\end{array}$ \\
\hline symbol & $d$ & $D$ & $L_{\text {free }} / N$ & $N$ & $L_{\text {free }}$ & $L_{\text {wire }} / N$ & $L_{\text {wire }}$ & $\alpha$ & $F_{d}$ & $F_{O}$ \\
\hline Units & $\mu \mathrm{m}$ & $\mu \mathrm{m}$ & $\mu \mathrm{m}$ & - & $\mu \mathrm{m}$ & $\mu \mathrm{m}$ & $\mu \mathrm{m}$ & degree & $\mathrm{THz}$ & $\mathrm{THz}$ \\
\hline $\begin{array}{l}\text { LH } \mu \text { coil-1 } \\
\text { LH } \mu \text { coil-2 } \\
\text { LH } \mu \text { coil-3 } \\
\text { LH } \mu \text { coil-4 } \\
\text { LH } \mu \text { coil-5 } \\
\text { RH } \mu \text { coil-1 } \\
\text { RH } \mu \text { coil-2 } \\
\text { RH } \mu \text { coil-3 } \\
\text { Straight wire } \\
\text { Freeze-dried } \\
\text { Spirulina }\end{array}$ & $\begin{array}{l}7 \\
7 \\
9 \\
7 \\
8 \\
8 \\
8 \\
6 \\
6 \\
5\end{array}$ & $\begin{array}{l}41 \\
26 \\
35 \\
22 \\
46 \\
30 \\
30 \\
33 \\
n / a \\
20\end{array}$ & $\begin{array}{c}77 \\
56 \\
27 \\
20 \\
16 \\
19 \\
14 \\
6 \\
\mathrm{n} / \mathrm{a} \\
44\end{array}$ & $\begin{array}{l}2.3 \\
4.5 \\
5.7 \\
5.6 \\
5.1 \\
6.8 \\
7.9 \\
7.0 \\
n / a \\
4.3\end{array}$ & $\begin{array}{r}174 \\
248 \\
153 \\
111 \\
81 \\
130 \\
110 \\
44 \\
320 \\
188\end{array}$ & $\begin{array}{r}150 \\
99 \\
113 \\
72 \\
145 \\
96 \\
95 \\
104 \\
n / a \\
77\end{array}$ & $\begin{array}{l}339 \\
441 \\
642 \\
399 \\
736 \\
658 \\
749 \\
725 \\
320 \\
328\end{array}$ & $\begin{array}{c}30.9 \\
34.0 \\
13.8 \\
16.1 \\
6.3 \\
11.4 \\
8.5 \\
3.5 \\
90 \\
35\end{array}$ & $\begin{array}{c}0.67-1.33 \\
1.02-2.03 \\
0.88-1.77 \\
1.39-2.78 \\
0.69-1.38 \\
1.04-2.08 \\
1.05-2.10 \\
0.96-1.93 \\
n / a \\
1.30-2.61\end{array}$ & $\begin{array}{c}0.5-1.5 \\
0.5-1.6 \\
0.5-1.9 \\
0.5-2.1 \\
0.4-1.2 \\
0.4-1.6 \\
0.3-2.0 \\
n / a \\
n / a \\
n / a\end{array}$ \\
\hline
\end{tabular}



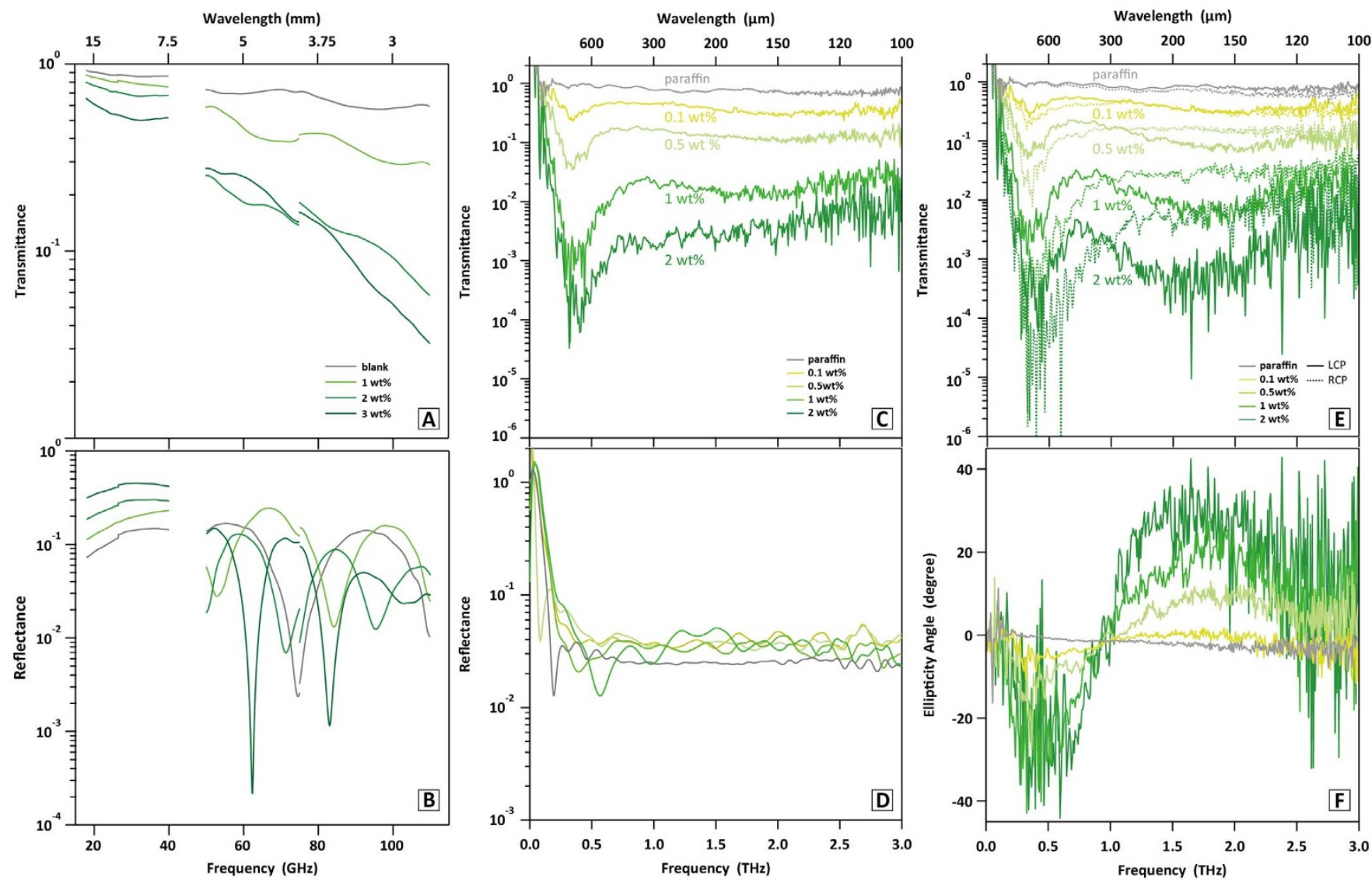

Figure 4 Electromagnetic response of copper $\mu$ coil sheets. (a), (b) Transmission and reflection spectra in the region of 18-40 GHz and 50-110 GHz with a prescribed amount of $\mu$ coils in paraffin and silicon matrix, respectively. (c), (d) THz transmission and reflection spectra of LH $\mu$ coil-1 using non-polarization mode. The concentration dependence was evaluated with four different wt $\%(0.1,0.5,1$, and 2 wt $\%$, colored with pale to dark green). (e), (f) THz transmission spectra under circular polarization mode and their ellipticity angle spectra. Those of a $\mu$ coil-free paraffin sheet (gray line) are also depicted as reference. LCP (solid line) and RCP (dotted line) show transmittances against left-handed and right-handed circular polarizations, respectively.

concentration change in the region of $\mathrm{V}$-band and $\mathrm{W}$-band (50 to $110 \mathrm{GHz}$ ), although multi-reflections based on the sheet thickness were observed in every sample. The sheet showed remarkable transmission loss possibly based on absorption, for example, less than $10 \%$ transmittance at $60 \mathrm{GHz}$ in case of $3 \mathrm{wt} \%$. A monotonous decrease in transmittance at higher frequency region led us to realize resonance specific to the $\mu$ coil structure in the region of $\mathrm{THz}$ wave. THz time-domain spectroscopy (THz-TDS) with nonpolarization mode was measured with the LH $\mu$ coil-1 dispersed into paraffin (Fig. 4c and 4d). No spectral feature with negligible reflection loss was observed in the $\mu$ coil-free paraffin sheet. Considerable transmission loss was observed over the entire region from 0.2 to $3.0 \mathrm{THz}$ for the paraffin sheets containing only small amount $(\sim 2 \mathrm{wt} \%)$ of the LH $\mu$ coil. The $\mu$ coil concentration showed linear relationship to optical density converted from the transmittance, which is consistent with Lambert-Beer law (Fig. S19). The concentration dependence proved no anomalous radiation from imperfect dispersibility of $\mu$ coils. Furthermore, reflectance of the sheet was around $3 \%$, so that the transmission loss was attributed to absorption of $\mathrm{THz}$ wave (for the spectra of all the $\mathrm{LH}$ $\mu$ coils with non-polarization mode, see Fig. S20). In order to evaluate optical activity of $\mu$ coil, THz-TDS combined with polarimetric analysis (THz-TDS-PA, SI-VII) ${ }^{16,17}$ was conducted with the same sample, LH $\mu$ coil-1. Significant difference was found in transmittances against RH and $\mathrm{LH}$ circular polarizations (Fig 4e). The spectrum of ellipticity angle gave negative and positive bands in around 0.5 and $1.5 \mathrm{THz}$, respectively (Fig. 4f). The spectroscopic evidences supported that the $\mu$ coil-dispersion sheet exhibits optically active $\mathrm{THz}$ response. Here, we considered function of the structure handedness of LH $\mu$ coil for the optical activity found in the isotropic dispersion, unlike metal helix array ${ }^{18,19}$. Chigrin et al. ${ }^{20}$ reported that achiral microparts can even effectively exhibit elliptical dichroism only based on their twisted configuration.

The $\mathrm{LH}$ and RH $\mu$ coils are optically active isomers. The variety of $\mu$ coils fabricated through systematic control of helical structures of Spirulina biotemplates enables us to experimentally evaluate structure-specific chiral electromagnetic responses. As references, it was confirmed that the dispersion sheets containing the straight copper wire and freeze-dried LH template- 2 showed optical inactive. All of LH $\mu$ coils gave the similar spectral features of their ellipticities as the LH $\mu$ coil-1, i.e., negative sign at $0.5 \mathrm{THz}$ and positive one above $1.0 \mathrm{THz}$ (Fig. 5a). At the same peak frequencies, the $\mathrm{RH}$ $\mu$ coils exhibited opposite signs of ellipticities (Fig. 5b). The LH $\mu$ coil-4 and RH $\mu$ coil-1 were selected here as enantiomeric pair with similar $L_{\text {free }} / N$ values. The ellipticities of the enantiomeric pair obviously showed mirror-image spectra with opposite signs; $+15^{\circ}$ for $\mathrm{LH} \mu$ coil- $4,-10^{\circ}$ for RH $\mu$ coil- 1 at $2.0 \mathrm{THz}$ (Fig. $5 \mathrm{c}$ ). Separately, another sheet containing a racemic mixture of $\mathrm{LH}$ and $\mathrm{RH} \mu$ coils with $1 \mathrm{wt} \%$ each traced the average of individual ellipticity angle spectrum, meaning no peaks with flat spectral feature (Fig. S21). The frequency showing sign inversion in ellipticity angle spectrum resulted in peak of rotation angle (Fig. S22). Therefore, it came to light that the LH $\mu$ coil emits the $\mathrm{RH}$ elliptical polarization and vice versa above the sign inversion frequency. That is to say, the LH $\mu$ coil shows dextrorotation and the RH one does levorotation. 

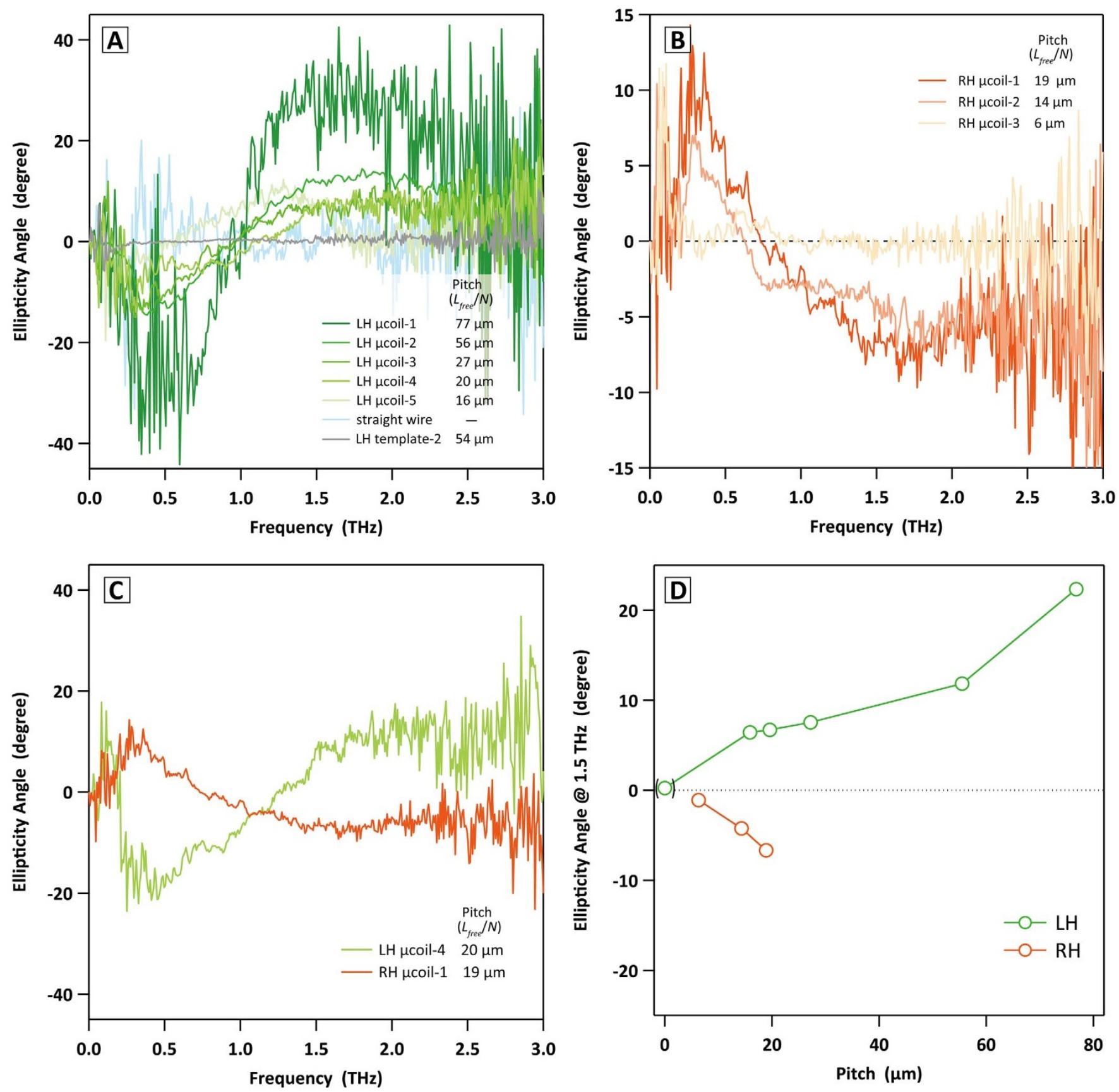

Figure $5 \mid$ Dependence of helical shape and handedness of $\mu$ coil on optically active response. (a), (b) Ellipticity angle spectra of $\mu$ coil-dispersion paraffin sheets were summarized into series of LH $\mu$ coils and RH $\mu$ coils as well as reference samples. All of samples contained $2 \mathrm{wt} \%$ in the paraffin matrices. The coil numbers and their $L_{\text {free }} / N$ values are depicted in insets. The RH $\mu$ coil shows laevorotation with ellipticity angle of opposite sign against the LH case. The intensity of ellipticity angle decreased and also the inversion frequency, where occurs the sign inversion from negative to positive for LH $\mu$ coil and opposite inversion for RH $\mu$ coil, shifted toward lower frequency region as the $L_{\text {free }} / N$ became smaller. Dispersion sheets of straight copper wire and freezedried LH template-2 with $54 \mu \mathrm{m}$ in $L_{\text {free }} / N$ showed no spectral features, which ensures the optical activity specific to the metal helical microstructure. (c) Ellipticity angle spectra of LH $\mu$ coil- 4 and RH $\mu$ coil-1 selected as enantiomeric pair. (d) Ellipticity angle at $1.5 \mathrm{THz}$ as a function of $L_{\text {free }} / N$.

The value from the straight copper wire was depicted with parenthesis at $0 \mu \mathrm{m}$ in pitch.

\section{Discussion}

There are three points of view to discuss the $\mathrm{THz}$ response observed in this study; the functional frequency region for $\mu$ coil response, the frequency at $0^{\circ}$ of ellipticity angle (sign inversion frequency), the degree of ellipticity angle. First, the peak of ellipticity angle at higher frequency showed a tendency to shift inversely proportionally to length of wire to make one pitch, $L_{\text {wire }} / N$. The relationship between the $L_{\text {wire }} / N$ and the functional frequency has been well explained by helical antenna theory ${ }^{21}$ and helix array system ${ }^{22}$, whose resonant wavelength is defined as $L_{\text {wire }} / N<\lambda<2 L_{\text {wire }} / N$. Briefly, it can be said that the $\mu$ coil sheet operates in a half-wave resonance mode $(\lambda=$ $\left.2 L_{\text {wire }} / N\right)$ (Table 1 and SI-VIII). In the lower frequency region, the correlation specific to the structure was not observed. The possible reason is that there are many other resonance modes such as dipole mode, internal resonance based on $L_{\text {free }} / N$, and inter-coil coupling. Second, the sign inversion frequency shifted toward lower frequency as the sample was varied from loose to tight $\mu$ coils, which appeared closely related to the $\alpha$. The sign inversion shift observed here was 
found as having dominant self-resonant frequency, $f_{0}$, expressed with inductance, $L$, and capacitance, $C$, i.e., $f_{0}=\frac{1}{2 \pi \sqrt{L C}}$. Since the tight $\mu$ coil has the shorter $L_{\text {free }} / N$ as well as the larger $N$ and/or $D$, the $L C$ value should be larger, consequently giving the lower $f_{0}$. Finally, as a crucial sense, the intensities of ellipticities decreased as the $L_{\text {free }} / N$ became smaller, in common with both of LH and RH $\mu$ coils (Fig. 5d). It has been reported that the helix array theoretically shows the same phenomenon ${ }^{22}$. In an extreme case, a ring or wire shape having $L_{\text {free }} /$ $N=0$ or $\alpha=90^{\circ}$, respectively, loses chirality. We are also considering a similarity between the $\mathrm{THz}$ chiral behavior of $\mu$ coil and exciton chirality of optically active molecule. Similar to Davydov splitting in molecular crystal, an interactive pair of dipole moments induced by electric or magnetic field change along the helix causes the resonance energy split into two. Due to their twisted configuration, both resonances are allowed and act asymmetrically electromagnetic induction, resulting in the ellipticities with opposite signs. Further discussion on the optical activity with helical antenna response is under investigation. The alignment control in the sheet may allow us to fully understand the $\mathrm{THz}$ responses in our system.

The biotemplating process was successfully demonstrated to develop a new class of 3D-structured material. The Spirulina-based $\mu$ coil exhibited the optical activity, of which the conditions such as the sense of wave rotation and the operation frequency were controlled by the structural parameters of $\mu$ coils. A wide distribution on the structural parameters, always attendant on the biotemplating process, would be rather effective for broadband operation. The present fabrication process can be applied for various distinctive biological tissues and microorganism with mass-productivity and a wide variety of material form, which will promise new strategy for material design.

\section{Methods}

Spirulina cultivation and shape control. The LH Spirulina (NIES-39 and -46, stock strains in National Institute for Environmental Studies) was cultivated with conventional aqueous media ${ }^{10}$ in an open-air water tank at room temperature under fluorescent light $(2,500 \mathrm{~lx})$. For the shape control of Spirulina template, the cultivation temperature and light intensity were raised up to $35^{\circ} \mathrm{C}$ and 7,500 $\mathrm{lx}$, respectively. The LH template- 1 was obtained from the standard condition and the LH template- 2 to -5 were collected every fourth day from the mass cultivation medium with the controlled condition under way into the tightening of helix, decreasing of $L_{f r e e} / N$. The linear strain was prepared by pure cultivation of the single trichome occurred by longer cultivation at the constant condition for more than 2 months.

Biotemplate process. The Spirulina was collected by a nylon mesh filter with 355 mesh and resuspended into a $100 \mathrm{~mL}$ of phosphate buffer solution $(\mathrm{pH}=7.0)$ including $4 \%$ glutaraldehyde as tissue fixation solution. The optical density of the Spirulina suspension was adjusted to be around 2.0 at $550 \mathrm{~nm}$ with an optical pass length of $1 \mathrm{~cm}$, which closely equals to the order of $10^{5}$ in the number of Spirulina per $1 \mathrm{~mL}$. The suspensions were left in ambient atmosphere overnight to complete fixation of Spirulina tissues. For further storage, they are kept in cool dark place so that the Spirulina can preserve their shapes and be used as the biotemplates for several years. Copper electroless plating onto the surface of Spirulina was conducted by the use of commercial products from Okuno Chemical Industries Co., Ltd.: for delipidation treatment, OPC-370 Condiclean MA; for Pd catalyzation (adsorption of Pd chloride based alkaline ionic catalyst), OPC-50 Inducer; for activation of the catalyst (reduction of Pd ion by dimethylamine borane), OPC-150 Cryster MU; for copper electroless plating, OIC Copper. All kinds of plating baths were initially prepared for $1 \mathrm{~L}$. The preparation of plating baths and each processing time were listed in Table S1. A $20 \mathrm{~mL}$ of fixed Spirulina suspension was filtered with the nylon mesh to collect the number of Spirulina with $2 \times 10^{6}$, corresponding to $12 \mathrm{mg}$ in weight of dried Spirulina and $200 \mathrm{~cm}^{2} / \mathrm{L}$ in bath load. It is noted that the surface area of one Spirulina is typically around $1 \times 10^{4} \mathrm{~cm}^{2}$. The Spirulina collected on the filter was immediately added to the baths before started to dry and stirred by mechanical stirrer with $300 \mathrm{rpm}$. The filtration and rinsing with water were successively carried out to switch the bath. After the final step with OIC Copper, the resulting copper $\mu$ coils were washed well on the filter and dispersed into $100 \mathrm{~mL}$ of distilled water overnight. The $\mu$ coils were collected by vacuum filtration using membrane filter $(0.8$ $\mu \mathrm{m}$ pore size, ATTP type, Isopore ${ }^{\mathrm{TM}}$ Track-Etched Membrane Filters) and dried in the atmosphere. The amount of $\mu$ coil generally averaged $90 \mathrm{mg}$, which gave $80 \%$ yield in the case of $\mu$ coil having $550 \mathrm{~nm}$ in the thickness of copper layer.

1. Bensaude-Vincent, B., Arribart, H., Bouligand, Y. \& Sanchez, C. Chemists and the school of nature. New J. Chem. 1, 1-5 (2002).
2. Sanchez, C., Arribart, H., Madeleine, M. \& Guille, G. Biomimetism and bioinspiration as tools for the design of innovative materials and systems. Nature Materials 4, 277-288 (2005).

3. Jin, M. H. et al. Superhydrophobic aligned polystyrene nanotube films with high adhesive force. Adv. Mater. 17, 1977-1981 (2005).

4. Ishii, D., Yabu, H. \& Shimomura, M. Novel biomimetic surface based on a selforganized metal-polymer hybrid structure. Chem. Mater. 9, 1799-1801 (2009)

5. Kamata, K. et al. Fabrication of left-handed metal microcoil from spiral vessel of vascular plant. Adv. Mater. 23, 5509-5513 (2011).

6. Vonshak, A. Spirulina Platensis Arthrospira: Physiology, Cell-Biology And Biotechnology (CRC Press, Bristol, PA, 1997).

7. Baurain, D. et al. Remarkable conservation of internally transcribed spacer sequences of Arthrospira ("Spirulina") (cyanophyceae, cyanobacteria) strains from four continents and of recent and 30-year-old dried samples from Africa. J. Phycol. 38, 384-393 (2002).

8. Zafaralla, M. T. et al. Protein from Spirulina. NSTA Technol. J. 10, 18-26 (1985)

9. Chen, B., Zhan, T., Lian, Z. \& Zhang, D. Magnetization of microorganism cells by sol-gel method. Sci. in China Ser. E: Technol. Sci. 51, 591-597 (2008).

10. Ogawa, T. \& Terui, G. Growth kinetics of Spirulina platensis in autotrophic and mixotrophic cultures. Ferment. Technol. Today, Proc. Int. Ferment. Symp. 4th, 543-549 (1972)

11. Ma, Z. \& Gao, K. Photoregulation of morphological structure and its physiological relevance in the cyanobacterium Arthrospira (Spirulina) platensis. Planta 230, 329-337 (2009).

12. Ishii, D. et al. Pd-Promoted Ni-P Electroless Deposition on a Hydrogen-Bonded Molecular Surface of a Supramolecular Fibrous Template. Chem. Mater. 18, 2152-2158 (2006).

13. Bockris, J. O. M., Reddy, A. K. N. \& Gamboa-Aldeco, M. E. Modern Electrochemistry 2A: Fundamentals of Electrodics, Electrodics Ch. 7, 1035-1400 (Kluwer Academic/Plenum Publishers, 2000).

14. Migneault, I., Dartiguenave, C., Bertrand, M. J. \& Waldron, K. C. Glutaraldehyde: behavior in aqueous solution, reaction with proteins, and application to enzyme crosslinking. BioTechniques 37, 790-802 (2004).

15. Mühling, M., Harris, N., Belay, A. \& Whitton, B. A. Reversal of helix orientation in the vyanobacterium Arthrospira. J. Phycol. 39, 360-367 (2003).

16. Hangyo, M. \& Takano, K. Metamaterials for terahertz waves. Mater. Integr. 24, 13-20 (2011)

17. Hangyo, M., Tani, M. \& Nagashima, T. Terahertz time-domain spectroscopy of solids: a review. Int. J. Infrared millimeter Waves 26, 1661-1690 (2005).

18. Gansel, J. K. et al. Gold helix photonic metamaterial as broadband circular polarizer. Science 325, 1513-1515 (2009).

19. Yu, Y., Yang, Z., Zhao, M. \& Lu, P. Broadband optical circular polarizations in the terahertz region using helical metamaterials. J. Opt. 13, 055104 (2011).

20. Chigrin, D. N., Kremers, C. \& Zhukovsky, S. V. Plasmonic nanoparticle monomers and dimers: from nanoantennas to chiral metamaterials. Appl. Phys. B 105, 81-97 (2011).

21. Balanis, C. A. Antenna Theory: Analysis and Design (Wiley, New Jersey, 2005).

22. Li, S., Yang, Z. Y., Wang, J. \& Zhao, M. Broadband terahertz circular polarizers with single- and double-helical array metamaterials. J. Opt. Soc. Am. A 28, 19-23 (2011).

\section{Acknowledgments}

This work was partly supported by MEXT KAKENHI, Grant-in-Aid for Scientific Research on Innovative Areas (22109006) and for Challenging Exploratory Research (22651051). Okuno Chemical Industries Co., LTD. provided electroless plating baths. The authors acknowledge Y. Akimoto and S. Hirashima for their support on SEM observations, and T. Nojima for helpful discussions. K.K. thanks T. Tadokoro for his invaluable comments about definition of circular polarization.

\section{Author contributions}

K.K., S.S., A.Y. and T.I. started this project; T.F. and W.T. contributed to the design of experiment; Z.P., M.I., S.H. and A.B. conducted Spirulina cultivation; K.T. and M.H performed the THz-TDS and data analysis; T.I. and M.H. coordinated the study with contributions from T.H., T.T. and K.N.; K.K. wrote the manuscript.

\section{Additional information}

Supplementary information accompanies this paper at http://www.nature.com/ scientificreports

Competing financial interests: The authors declare no competing financial interests. How to cite this article: Kamata, K. et al. Spirulina-Templated Metal Microcoils with Controlled Helical Structures for THz Electromagnetic Responses. Sci. Rep. 4, 4919; DOI:10.1038/srep04919 (2014)

This work is licensed under a Creative Commons Attribution 3.0 Unported License. The images in this article are included in the article's Creative Commons license, unless indicated otherwise in the image credit; if the image is not included under the Creative Commons license, users will need to obtain permission from the license holder in order to reproduce the image. To view a copy of this license, visit http://creativecommons.org/licenses/by/3.0/ 